\title{
Italian Society of Arthroscopy (SIA) join Muscles, Ligaments and Tendons Journal MLTJ
}

\author{
Nicola Maffulli ${ }^{1}$ \\ Piero Volpi ${ }^{2}$ \\ 1 Department of Musculoskeletal Disorders \\ Faculty of Medicine, Surgery and Dentistry, \\ University of Salerno, Salerno, Italy; \\ Centre for Sports and Exercise Medicine \\ Barts and London School of Medicine and Dentistry, \\ Queen Mary University of London, London, UK \\ 2 Knee Orthopaedic and Sports Traumatology Unit, \\ Humanitas Research Hospital, Rozzano, Italy
}

With this number, yet another venture takes shape. When Muscles Ligaments and Tendons Journal (MLTJ) started, it was just a bet: it was to be the scientific organ of the Italian Society of Muscles Ligaments and Tendons (ISMuLT). It was a small journal, and was to rely on the contribution of the superspecialists in this field. The tireless work of those around me, and the utmost quest for scientific quality, has produced a scientific journal that was able to be listed in PubMed within 18 months of its first issue, and to become really international: a glimpse at this issue will show that more than $50 \%$ of the published articles come from foreign institution.

With this issue, the adventure continues, and raises to yet another new exciting higher level. The association with the SIA (Italian Society of Arthroscopy) brought to fruition the work and the vision of Dr. Piero Volpi, the President of SIA. Dr. Volpi is a man of vision and intellect, and it is therefore with open arms that MLTJ welcomes the SIA.

To more, together.

Prof. Nicola Maffulli

President of Italian Society of Muscles, Ligaments \& Tendons (ISMuLT)
A major goal of a learned society is to promote and encourage the publication of studies that allow disseminating the knowledge and experience of experts in the specific field. The Società Italiana di Artroscopia - SIA (Italian Society of Arthroscopy) has always sought to collaborate with qualified journals for the publication of scientific papers. The knowledge update and the technical developments resulting from the participation in courses and congresses are important, but only the opportunity to write and publish scientific articles allows specialists to complete and enrich their professional background. Reading a scientific article entices and interests us all, but the scientific and cultural maturity that an Author obtains from formulating a hypothesis, collecting and analysing data, reviewing the literature, preparing, documenting, writing and polishing an article is unmatched in the path of continuing professional development required by our profession.

For this purpose, learned societies such as the SIA which want to transmit and publicise innovative and newly conceived surgical procedures, through its experts, need to consolidate the results achieved with these methods. Clarity of exposition, integrity of data and logics of writing are crucial to validate the hypotheses of their studies, just as references are needed to underscore, confirm and identify any differences. Therefore, providing the Orthopaedic, Arthroscopy and Sports Traumatology world with an international indexed journal with a good impact factor is certainly an added value. Muscles Ligaments and Tendons Journal (MLTJ), wanted, conceived and edited by Professor N. Maffulli, is an unique opportunity for the SIA to publish its scientific contributions. Young people looking for affirmation and greater professional growth through their work should take advantage of this collaboration between our Society and MLTJ to make a name for themselves in this difficult competitive scientific world.

\section{Dr. Piero Volpi}

President of the Italian Society of Arthroscopy (SIA) 